\title{
HARMONI: First light spectroscopy for the ELT: final design and assembly plan of the spectrographs
}

Ozer, Z., Schnetler, H., Bagci, F. T., Booth, M., Brock, M., et al.

Z. Ozer, H. Schnetler, F. T. Bagci, M. Booth, M. Brock, N. Cann, J. Capone, J. Chao Ortiz, G. Dalton, N. Dobson, T. Foster, A. Hidalgo Valadez, J. Kariuki, I. Lewis, A. Lowe, J. Lynn, M. Rodrigues, I. Tosh, F. Clarke, M. Tecza, N. Thatte, "HARMONI: First light spectroscopy for the ELT: final design and assembly plan of the spectrographs," Proc. SPIE 11447, Ground-based and Airborne Instrumentation for Astronomy VIII, 114472H (13 December 2020); doi: $10.1117 / 12.2560359$ 


\title{
HARMONI: First Light Spectroscopy For The ELT: Final Design and Assembly Plan of The Spectrographs
}

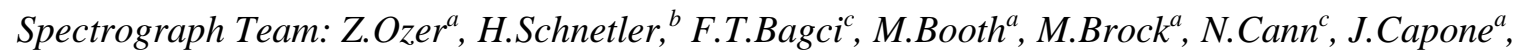 \\ J.Chao Ortiz, G.Dalton ${ }^{a}$, N.Dobson ${ }^{b}$, T.Foster ${ }^{a}$, A.Hidalgo Valadez ${ }^{a}$, J.Kariuki ${ }^{a}$, I.Lewis ${ }^{a}$, A.Lowe ${ }^{a}$, \\ J.Lynn ${ }^{a}$, M.Rodrigues ${ }^{a}$, and I.Tosh ${ }^{c}$ \\ Project Office: F.Clarke ${ }^{a}$, M.Tecza ${ }^{a}$, and N.Thatte ${ }^{a}$ \\ ${ }^{a}$ University of Oxford, Denys Wilkinson building, Keble Road OX1 3RH, Oxford, UK \\ ${ }^{b}$ Astronomy Technology Centre Royal Observatory, Edinburgh Blackford Hill, Edinburgh, UK \\ ${ }^{c}$ RAL Space, Rutherford Appleton Laboratory, Harwell Campus, Didcot, OX11 OQX, UK STFC UK
}

\begin{abstract}
HARMONI is the first light visible and near-IR integral field spectrograph for the ELT. It covers a large spectral range from $450 \mathrm{~nm}$ to $2450 \mathrm{~nm}$ with resolving powers from $\mathrm{R}(\equiv \lambda / \Delta \lambda) 3500$ to 18000 and spatial sampling from 60mas to 4mas. It can operate in two Adaptive Optics modes - SCAO (including a High Contrast capability) and LTAO - or with NOAO. The project is preparing for Final Design Reviews. The instrument uses a field splitter and image slicer to divide the field into 4 sub-units, each providing an input slit to one of four nearly identical spectrographs. This proceeding presents the final optomechanical design and the AIV plan of the spectrograph units.
\end{abstract}

\section{INTRODUCTION}

Four spectrograph units is the sub-system of HARMONI where imaging is combined with spectroscopy; the third dimension for the two-dimensional spatial data, carried by the fully reflective optical design of HARMONI, is created by dispersing the light in a range of spectral resolutions and wavelengths that can be selected by the user. Spectrograph sub-system is shown in the HARMONI product break-down structure in Figure 1.

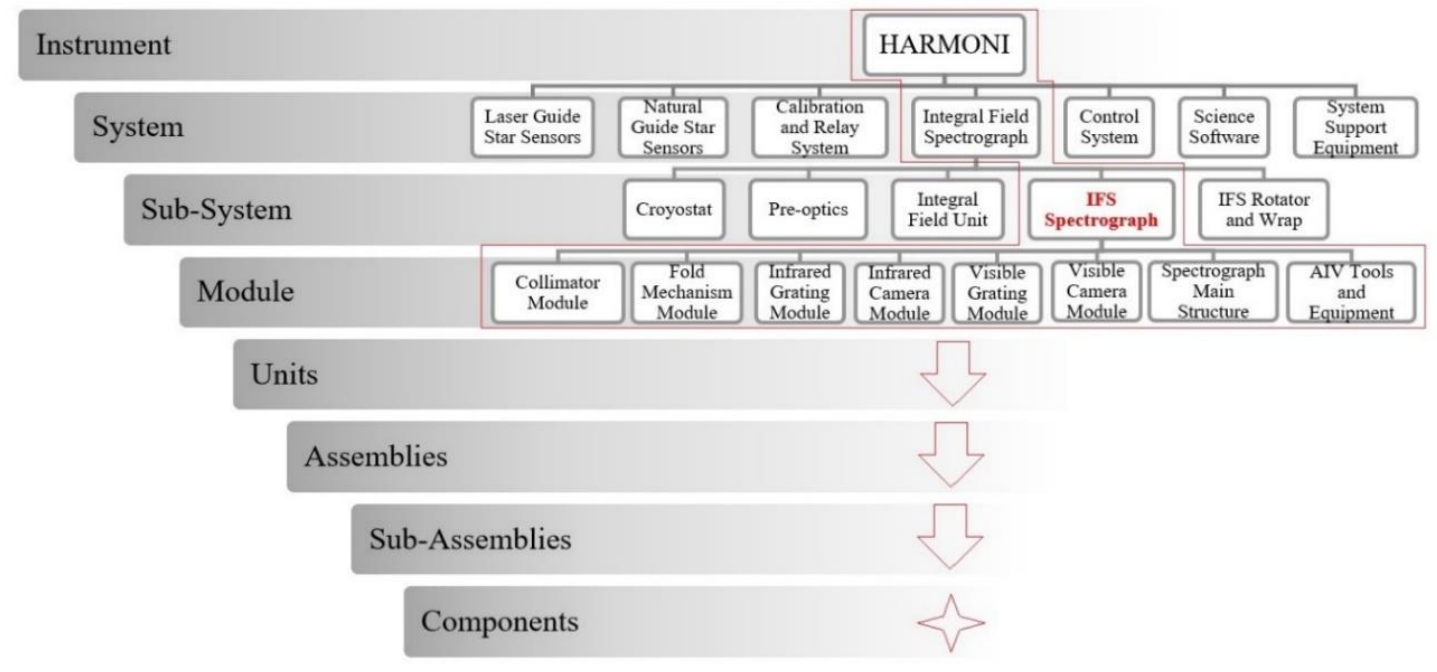

Figure 1. Spectrograph Sub-System In HARMONI Product Break-Down Structure

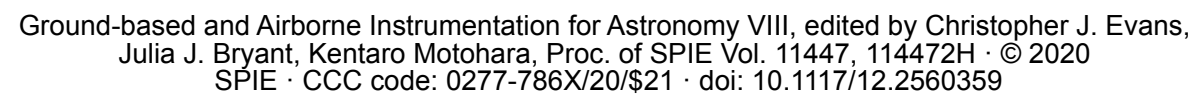


HARMONI successfully completed the preliminary design review (PDR) in December 2018 and moved to the final design phase. Final design reviews (FDR) will start with the requirements, continue with the optical design and systems, and finalize with the instrument. Upon completion of the system level FDR phase, all sub-systems will implement their Manufacturing, Assembly, Integration and Testing (MAIT) plans. The spectrographs, which will successfully complete their verification tests at the University of Oxford facilities, will be shipped to the integration site at the UK Astronomy Technology Centre in Edinburgh and the Assembly, Integration and Verification (AIV) process of the instrument will be performed there.

\section{DESIGN OF THE SPECTROGRAPH}

The spectrograph design allows minimal loss till the refractive optics, by conveying light with high reflectance optics through the instrument and dividing it into the visible and infrared channels at the very end. Also, since it will operate in a cryogenic environment $(130 \mathrm{~K})$, all requirements and tolerances have been determined accordingly.

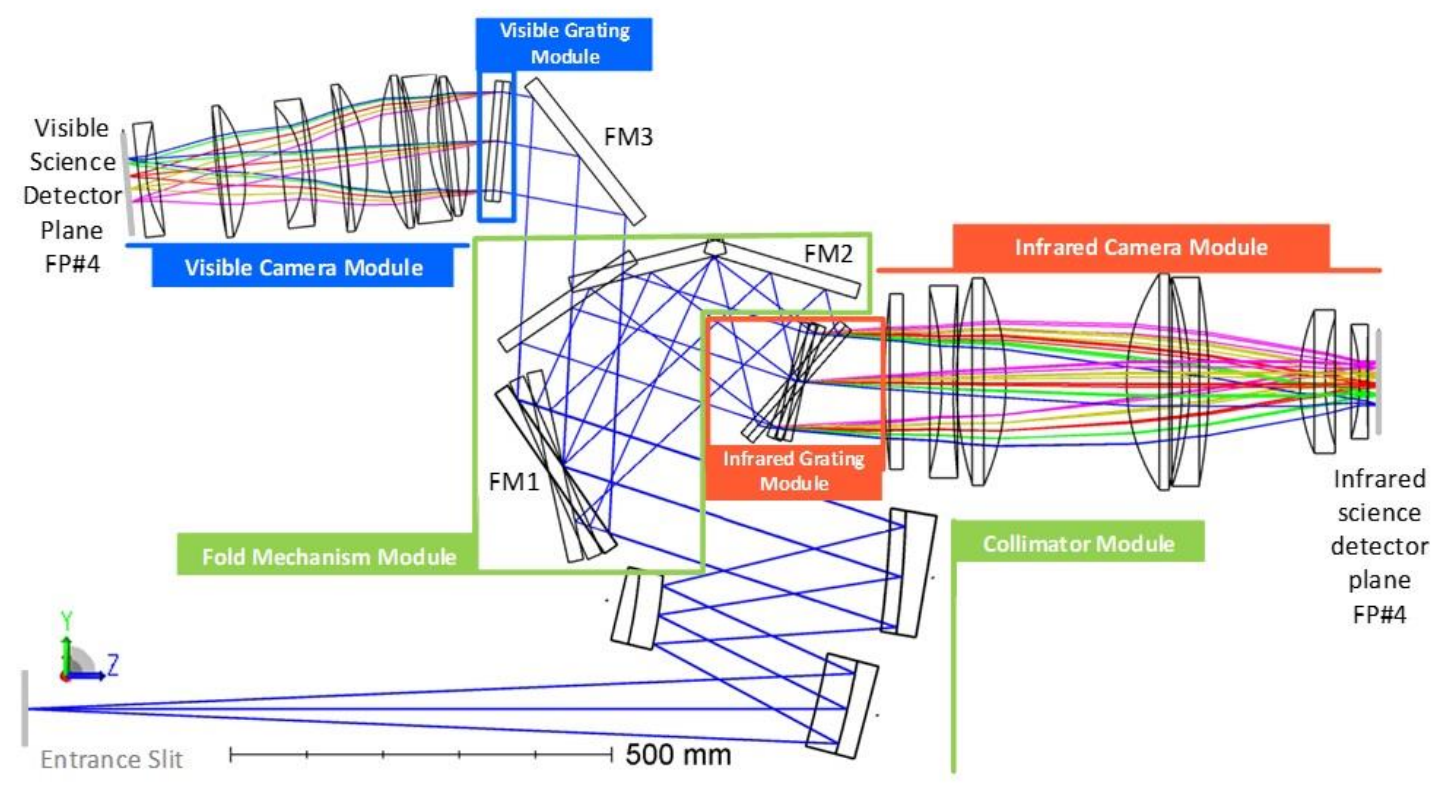

Figure 2. Optical Layout of The Spectrograph

Figure 2 shows the optical ray trace of the spectrograph. The collimator module guides the light from the entrance slit, that is formed by an image splitter and image slicer, and collimates it into the folding mechanism module (FMM) that provides the user with the ability to select wavelength ranges between visible and near-infrared. Main component of the FMM are the articulated fold mirror (FM1) and the swing-arm fold mirror (FM2), which direct the light into the visible or the near-infrared channels with the required input angles of Volume Phase Holographic gratings for each wavelength configuration. In the visible channel there is only one grating assembly that disperse visible light as an input for the 
visible camera module. On the other hand, there are eleven grating assemblies for different bands in the infrared grating module that is controlled with the help of the grating wheel drive and the grating wheel. The light is separated in the selected band with a specific spectral resolution through the infrared grating module and enters the infrared camera module. Both visible and infrared camera modules focus the dispersed light on to the science detector focal planes which are mosaics of two $4 \mathrm{Kx} 4 \mathrm{~K}$ detectors. The design has been made so that each spectrograph is the same, but due to the budget limitations, only two spectrographs will have visible channels.

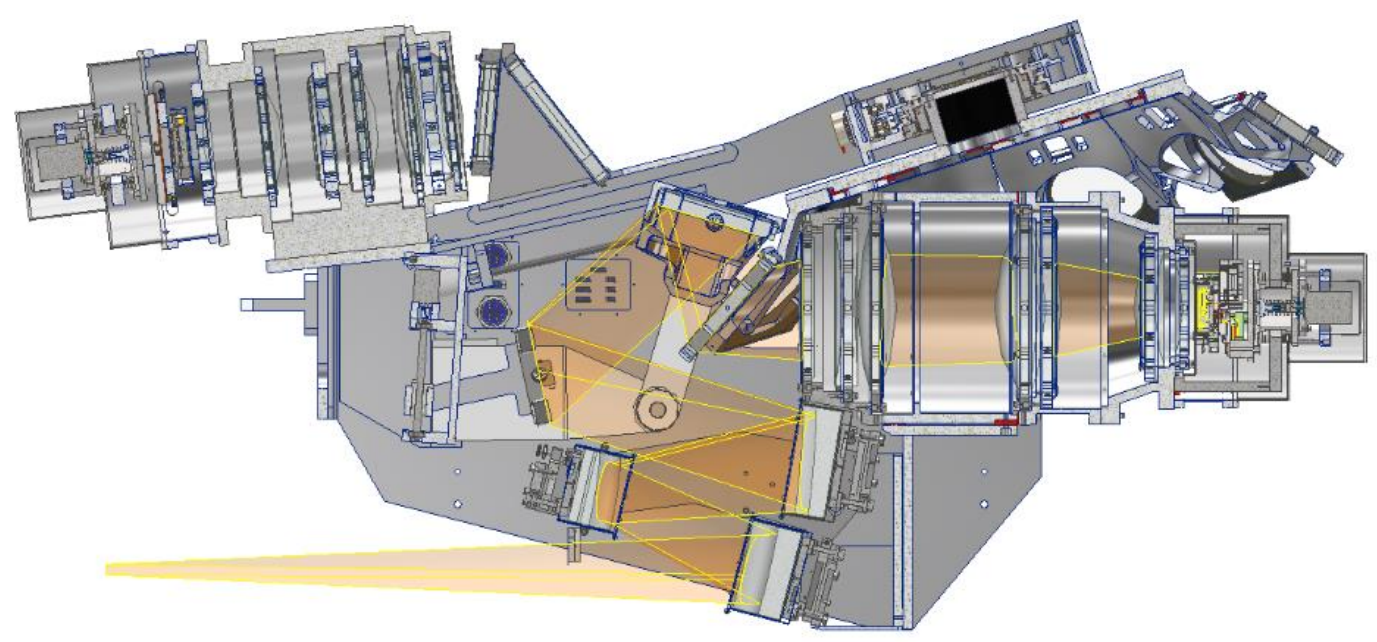

Figure 3. Mid-Section Mechanical Layout of The Spectrograph With Highlighted IR Channel

Currently, the optical design of the spectrograph has been completed and the tolerances are evaluated in line with the information obtained from the manufacturer companies and the instrument requirements that are driven by our scientific cases. The conceptual mechanical design has been completed and works on the detailed design are ongoing nd final version is given in Figure 3. These design works also include special tooling, equipment and storage units that will be used during the MAIT and AIV phases. A single spectrograph will be approximately [2190 x $1340 \times 990] \mathrm{mm}$ in size and $485 \mathrm{~kg}$ in weight. Therefore, it is necessary to carefully consider the transportation, lifting and movement requirements in various directions for all activities to be performed at every level, as well as testing and assembly operations.

\section{ASSEMBLY, INTEGRATION AND VERIFICATION PLAN OF THE SPECTROGRAPH}

The MAIT phase, which will start with the completion of the system level FDR, includes all the activities in the phase of establishing the manufacturability by making all procurements and completing the necessary quality control inspections, the assembly and integration by following the relevant procedures, and the verification of the produced with the relevant tests. This process also includes interim investigations, namely; Manufacturing Preparation, Integration Preparation and Acceptance 
Review which are supported by some evidences like test and inspection reports, compliancy matrices etc. Upon successful completion of the Acceptance Review for the relevant Sub-System, it is delivered for the System AIV process.

Until the FDR stage, it will be ensured that components of the design can be manufactured by making part-specific interviews with the subcontractor companies and the mechanical workshops in the facilities. After the tenders to be held, the most suitable sub-contractors will be determined among the participating companies according to their skills, experience, price and delivery schedule. This choice will support HARMONI's budget and master project schedule, while minimizing critical risks such as the quality of materials and compliance with the requirements. Thus, the manufacturability will be ensured by the delivery of the purchase orders and the completion of the quality control inspections.

Before the parts are assembled and verified, the consistency of the procedures and the equipment, tooling and infrastructure to be used for assembly, integration and tests will be verified with an engineering model of the spectrograph that does not include any optics. Although all processes have been planned with great consideration, it is deemed necessary to verify the processes as it would be involved with a much larger structure than the systems previously built.

When the necessary components for each module are completed the workflow that is given in Figure 4 will be followed. The assembly phase will start with the mechanical assembly till the module level and only functional checks will be provided. When it comes to the module level, the optical components will be included in the assembly stage and opto-mechanical modules will be produced. The mentioned modules (collimator module, fold mechanism module, infrared grating module, infrared camera module, visible grating module, visible camera module) will be checked first in a warm environment functionally, then in a cryogenic test chamber with a diameter of $1 \mathrm{~m}$ at $130 \mathrm{~K}$ temperature for verification.

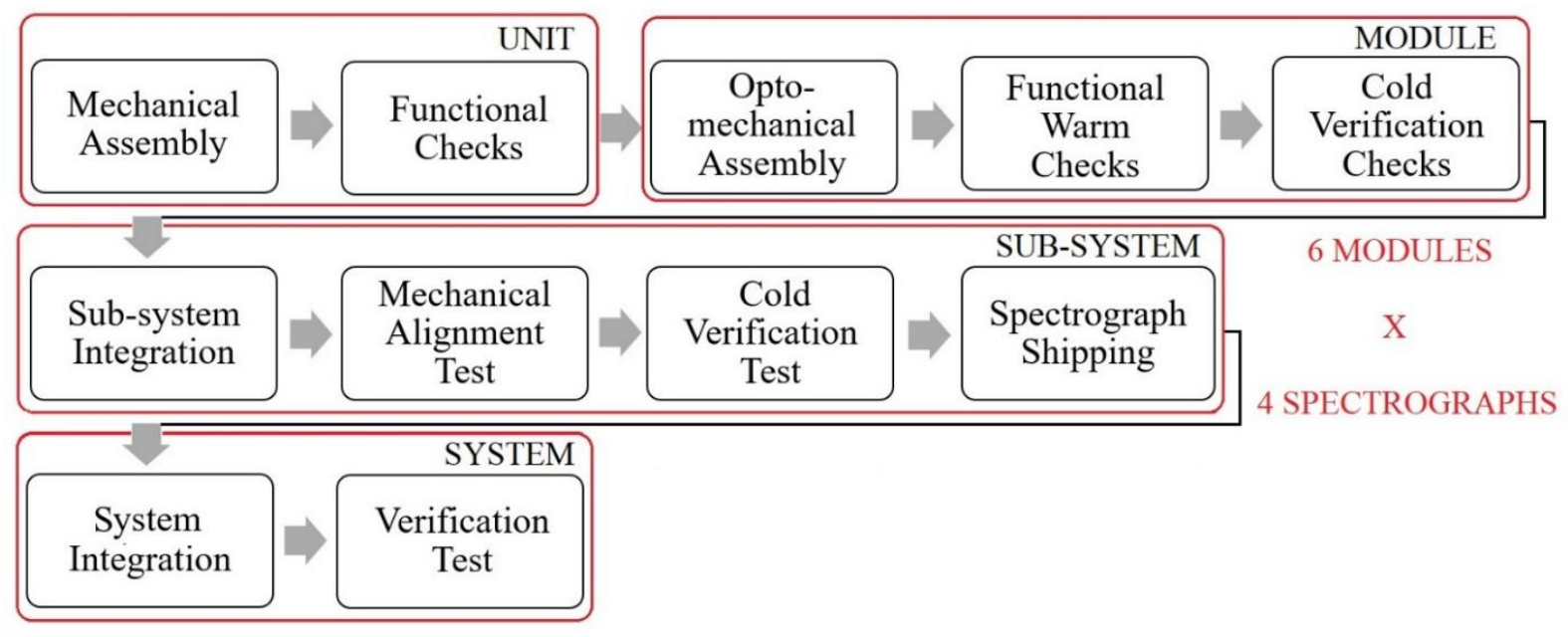

Figure 4. Flow Chart of AIV Phase 
After the verifications in warm and cold environments, the sub-system level integration phase will be started and the modules will be assembled to the spectrograph main structure with the help of AIV tools and equipment. The alignment to be made at this stage is of great importance in terms of meeting the system level requirements. After the mechanical alignment tests are successfully completed, the spectrograph subsystem will be tested in a cryogenic test chamber with a diameter of $2.2 \mathrm{~m}$ at $130 \mathrm{~K}$ temperature as shown in Figure 5. Thus, if there is a problem in the sub-system stage, since an operating temperature test is already performed in the module level, it will be detected that there is an error due to integration or alignment. Since there are many variables up to the sub-system level, checking after the assembly at every stage has been determined as the method required to identify and control the main source of the problems to be encountered during the production phase. Moreover, the engineering grade detectors will be used in science detector planes during the verification of alignment and cold tests, and in this way, the detector sub-systems that will be included in the final products will be protected. With the successful completion of the cold tests to be performed at the sub-system level, the first spectrograph with both visible and infrared channels, will be sent to the integration site in the UK Astronomy Technology Centre, Edinburgh where the system level AIV process will be carried out.

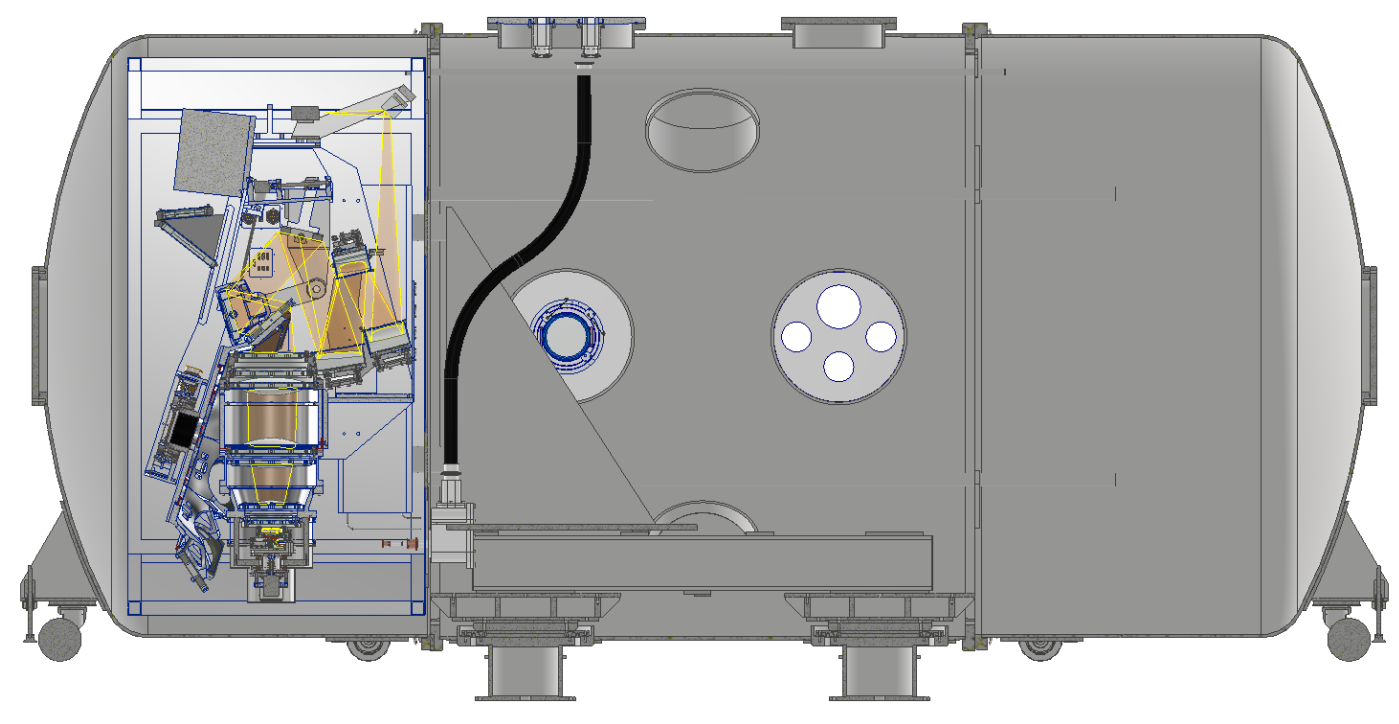

Figure 5. Spectrograph in the cryogenic test chamber with a diameter of $2.2 \mathrm{~m}$

The assembly, integration and testing of each spectrograph will take approximately 6 months and they will be integrated into the system level one by one, hence the production of the spectrographs will be performed in series. After the first spectrograph shipment, one spectrograph will be shipped every six months. Also, as the assembly and verification stages of opto-mechanic modules take a long time, the infrared camera module and visible camera module work packages are took on by RAL Space. The modules will be tested and delivered to the University of Oxford for the sub-system level integration phase. 
With the completion of deliveries to the integration site, due to limitations such as the size of the spectrographs, the space available in the facilities, and the schedule of system level integrations, it will be possible to test four spectrographs at the same time at the system level integration stage. For this reason, verification of the activities carried out at every stage, proper storage of the final products and their shipment under appropriate conditions are considered as a condition of not encountering a problem at the system level. After these verifications will be made, the AIVs of the four Spectrographs at the system level can be completed.

\section{FUTURE PLANS}

In the current situation, detailed design studies are formed in line with the feedbacks received from oneto-one interviews with the manufacturers and the responses obtained after the Request For Information phases conducted through the purchasing department. The maturation of the documents presented at the PDR stage in line with the information gained is in progress.

After the FDR of requirements and optical design to be realized in 2021, system level FDR will be occurred in the beginning of 2022 and purchasing processes will be started accordingly. Considering the length of the material procurement times, the time required to create manufacturability, and the assembly, integration and test times: the first spectrograph is aimed to be ready at the beginning of 2024 . 